\title{
Selbstreguliertes Lernen in unterschiedlichen Phasen des Bildungsverlaufs
}

\author{
Yves Karlen · Silke Hertel
}

(C) Springer Fachmedien Wiesbaden GmbH, ein Teil von Springer Nature 2018

Die Fähigkeit, das eigene Lernen selbst zu regulieren, wird zunehmend gefordert sei es in der Schule, im Studium oder in der Berufswelt. Sie umfasst verschiedene Selbstregulationsmaßnahmen auf kognitiver, metakognitiver, motivational-emotionaler oder verhaltensbezogener Ebene. Auf diese können Lernende zurückgreifen, um ihre mit dem Lernprozess verbundenen Gedanken, Emotionen und Handlungen sowie ihre Motivation und den Lernprozess selbst erfolgreich sowie zielgerichtet zu steuern und zu regulieren (Schiefele und Pekrun 1996; Zimmerman 2000). Neben der Kompetenz, kognitive, metakognitive und ressourcenbezogene Lernstrategien gezielt und mit einer hohen Anwendungsqualität einzusetzen, verfügen erfolgreiche selbstregulierte Lernende insbesondere über umfangreiches metakognitives Wissen und die Bereitschaft, Herausforderungen anzunehmen (Pressley et al. 1989). Diese Selbstregulationskompetenzen nehmen vor dem Hintergrund des lebenslangen Lernens eine besondere Rolle ein, da sie in einem positivem Zusammenhang mit schulischem und außerschulischen Erfolg stehen (Dent und Koenka 2016; Dignath und Büttner 2008; Zimmerman und Schunk 2011). Spezifische Kompetenzdimensionen des selbstregulierten Lernens zeigen höhere Zusammenhänge zu akademischem Erfolg als beispielsweise Intelligenz oder sozioökonomischer Status (Richardson et al. 2012). Allerdings weisen Studien darauf hin, dass diese Kompetenzen auch bei Studierenden teilweise nur gering ausprägt sind. Lernende unterschiedlicher Altersstufen sehen in der Selbstregulation ihrer Lernprozesse eine große Herausfor-

\footnotetext{
Y. Karlen $(\bowtie)$

Institut Sekundarstufe I \& II, Pädagogische Hochschule FHNW, Bahnhofstrasse 6, 5210 Windisch, Schweiz

E-Mail: yves.karlen@fhnw.ch

S. Hertel

Institut für Bildungswissenschaft, Ruprecht-Karls-Universität Heidelberg,

Akademiestraße 3, 69117 Heidelberg, Deutschland

E-Mail: hertel@ibw.uni-heidelberg.de
} 
derung (McCabe 2011; Peverly et al. 2003). Zugleich bringen sie unterschiedliche personenbezogene Voraussetzungen (u.a. Persönlichkeitseigenschaften, motivationale Dispositionen, vorhandene Kompetenzen) mit, die einen eher hemmenden oder eher begünstigenden Einfluss auf das selbstregulierte Lernen haben können (z. B. Bidjerano und Dai 2007; Wolters und Hussain 2015). Der Einsatz von Selbstregulationskompetenzen wird neben diesen internalen Faktoren auch von externalen Faktoren (u. a. Lernsituation, Aufgabe), die die Anforderungen an den Strategieeinsatz modulieren können, bestimmt (z. B. Engelschalk et al. 2016; Malmberg et al. 2013). Selbstreguliertes Lernen wird folglich stets von den individuellen Voraussetzungen und vom Lernkontext beeinflusst.

Vor diesem Hintergrund gewinnen der Erwerb und die Vermittlung von Kompetenzen des selbstregulierten Lernens in verschiedenen Phasen des Bildungsverlaufs sowie in unterschiedlichen formalen Bildungsinstitutionen an Bedeutung. Die bisherige Forschung hat gezeigt, dass sich Kompetenzen im selbstregulierten Lernen durch gezielte Trainingsmaßnahmen über verschiedene Altersstufen hinweg erfolgreich fördern lassen (Donker et al. 2014; Hattie et al. 1996). Die Förderung kann sowohl implizit durch die didaktische Gestaltung von offenen Lernumgebungen und geeigneten Lernaufgaben als auch explizit über die direkte Vermittlung von Strategien und Wissen zum selbstregulierten Lernen im Unterricht, in Lehrveranstaltungen oder in Trainingsprogrammen erfolgen (z. B. Moos und Ringdal 2012; Paris und Paris 2001; Perry und VandeKamp 2000). Zudem stellt sich die Frage, welche Schülerinnen und Schüler vor dem Hintergrund ihrer individuellen Voraussetzungen (z. B. Alter, Migrationshintergrund, sozio-ökonomischer Status, kognitive Fähigkeiten, motivationale Orientierungen) von Fördermaßnahmen zur Vermittlung von Selbstregulationskompetenzen in besonderem Maße profitieren (Donker et al. 2014; Sontag und Stoeger 2015; Stebner et al. im Druck).

Die vier Beiträge in diesem Thementeil befassen sich mit dem selbstregulierten Lernen in unterschiedlichen Abschnitten der Bildungskarriere und nehmen hierbei verschiedene Perspektiven ein. Die ersten beiden Beiträge untersuchen das selbstregulierte Lernen am Ende der Grundschulzeit (Benick et al., dieses Heft) sowie in der Sekundarstufe I (Schuster et al., dieses Heft) und beschreiben Effekte von direkten und indirekten Unterstützungs- und Trainingsansätzen. Sie gehen den Fragen nach, ob Schülerinnen und Schüler mit unterschiedlichen Kompetenzprofilen im selbstregulierten Lernen mehr oder weniger von einem Training profitieren (Benick et al., dieses Heft) und inwiefern Schülerinnen und Schüler die im Rahmen von Trainingsprogrammen zu selbstreguliertem Lernen erworbenen Kompetenzen erfolgreich auf Aufgabenstellungen im Schulalltag transferieren können (Schuster et al., dieses Heft). Die beiden weiteren Beiträge nehmen die Sekundarstufe II (Karlen et al., dieses Heft) sowie das Studium an der Hochschule (Bäulke et al., dieses Heft) in den Blick und betrachten die Bedeutung unterschiedlicher Aspekte des selbstregulierten Lernens für den Lernerfolg bei einer komplexen Aufgabenstellung (Karlen et al., dieses Heft) sowie für den Verbleib im Studium (Bäulke et al., dieses Heft). Sie nehmen auch das Zusammenspiel von verschiedenen Selbstregulationskompetenzen und internalen Faktoren in den Blick. 
Der erste Beitrag von Benick et al. untersucht in einem ersten Schritt, welche Selbstregulationsprofile bei Viertklässlerinnen und Viertklässlern vorliegen. Mittels latenter Profilanalyse konnten vier Selbstregulationsprofile identifiziert werden, die sich in metakognitiven und motivationalen Kompetenzdimensionen des selbstregulierten Lernens (z. B. Zielsetzung, Zeitplanung, Selbstmotivation) voneinander unterscheiden. In einem zweiten Schritt wurde analysiert, ob und inwiefern Schülerinnen und Schüler mit entsprechend unterschiedlicher Ausgangslage im selbstregulierten Lernen von einer fächerübergreifenden Intervention zur Vermittlung und Förderung selbstregulativer Strategien profitieren. Die Intervention wurde prozessual auf Basis von Lerntagebuchdaten auf ihre spezifische Wirksamkeit hin verschiedenen Selbstregulationsprofilen auch unterschiedlich von den Interventionseinheiten profitieren. Der Beitrag verdeutlicht, dass die Intervention zur Förderung des selbstregulierten Lernens differentielle Effekte aufweist (u. a. finden sich für Schülerinnen und Schüler mit niedrigen Ausprägungen im selbstregulierten Lernen die höchsten Interventionseffekte), die bei der Konzeption zukünftiger Trainings berücksichtigt werden sollten.

Der zweite Beitrag von Schuster et al. überprüft, ob die in einem kombiniertem Training zum selbstregulierten Lernen - bestehend aus einem direktem Training außerhalb des Schulunterrichts und einem indirekten Training im regulären Schulunterricht - erlernten Selbstregulationskompetenzen von Schülerinnen und Schüler auf nahe und ferne Lernaufgaben transferiert werden können. Hierzu wurden die Anwendung kognitiver und metakognitiver Strategien beim Bearbeiten einer Lernaufgabe im Training (naher Transfer) und im Deutschunterricht (ferner Transfer) von Schülerinnen und Schülern der 5. und 6. Jahrgangsstufe mittels eines situationsspezifischen Vignettentests erfasst. Anhand eines quasi-experimentellen Untersuchungsdesigns mit Experimental- (zeitgleich ein direktes und indirektes Training) und Kontrollgruppe (kein Training) wurden die Transfereffekte untersucht. Die Ergebnisse zeigen einen Vorteil der Experimentalgruppe beim Lösen einer nahen Transferaufgabe sowohl in Bezug auf kognitive als auch auf metakognitive Strategien. Zudem schnitt die Experimentalgruppe beim Bearbeiten einer fernen Transferaufgabe in Bezug auf die metakognitiven Strategien besser ab. Insgesamt macht die Studie deutlich, dass es mit einem kombinierten Training gelingen kann, Transfereffekte zu erzielen.

Der dritte Beitrag von Karlen et al. fokussiert auf den Zusammenhang zwischen Grit (Durchhaltevermögen), unterschiedlichen Selbstregulationskompetenzen und der Leistung bei der Maturaarbeit, einer längerfristigen, anspruchsvollen schriftlichen Arbeit am Ende der gymnasialen Oberstufe. Grit, kognitive und motivationale Regulationskompetenzen wurden anhand eines Fragebogens erhoben. Das konditionale Wissen zu motivationalen Regulationsstrategien, sogenanntes metamotivationales Wissen, wurde mit einem situationsspezifischen Wissenstest erfasst. Die Befragung verdeutlicht, dass die beiden Grit Dimensionen „Beharrlichkeit“ und „Beständiges Interesse“ unterschiedliche Prädiktionskraft für Kompetenzen im selbstregulierten Lernen aufweisen. Beharrlichere Gymnasiastinnen und Gymnasiasten gaben an, vermehrt motivationale Regulationsstrategien zu nutzen, verfügten über höheres metamotivationales Wissen und waren stärker davon überzeugt, über höhere kognitive Regulationskompetenzen zu verfügen als Gymnasiastinnen und Gymnasiasten, 
die weniger beharrlich waren. Beständiges Interesse hatte einen positiven Effekt auf die kognitiven Regulationskompetenzen und war negativ mit motivationalen Regulationsstrategien korreliert. Die Beharrlichkeit sagte zusätzlich zu den kognitiven Regulationskompetenzen die Maturaarbeitsnote vorher. Diese Studie verdeutlicht, dass bei einer anspruchsvollen und längerfristigen Lerntätigkeit sowohl Grit als auch Selbstregulationskompetenzen zum Lernerfolg beitragen.

Der vierte Beitrag von Bäulke et al. befasst sich mit Fragen nach den Zusammenhängen zwischen dem metakognitiven Wissen zu motivationalen Regulationsstrategien, der Effektivität der Motivationsregulation, der akademischen Prokrastination und den Studienabbruchsintentionen von Studierenden. Die Effektivität der Motivationsregulation, die akademische Prokrastination sowie die Studienabbruchintention wurden anhand von Selbstberichten erfasst. Zusätzlich wurde ein situationsspezifischer Wissenstest zur Erfassung des metakognitiven Wissens verwendet. Die Ergebnisse zeigen, dass das konditionale Motivationsregulationsstrategiewissen einen direkten, positiven Effekt auf die Effektivität von motivationalen Regulationsstrategien sowie einen indirekten negativen Effekt auf die Prokrastination und die Studienabbruchintension hat. Die Effektivität der Motivationsregulation korreliert negativ mit der akademischen Prokrastination und mit der Intention, das Studium abzubrechen. Diese Ergebnisse machen deutlich, dass Kompetenzen der motivationalen Regulation wichtige Resilienzfaktoren zur Verhinderung von Prokrastination und von Studienabbruch darstellen.

Die vier Beiträge in diesem Thementeil eröffnen somit eine breite Perspektive auf das selbstregulierte Lernen: Sie nehmen es als abhängige Variable und als unabhängige Variable in den Blick, thematisieren das Zusammenspiel von selbstreguliertem Lernen mit weiteren individuellen Lernvoraussetzungen und beschreiben Transfereffekte im Alltag von Lernenden. Dabei verwenden die Autorengruppen vielfältige methodische Zugänge, die von Fragebögen über Wissenstests bis hin zu der Erfassung von Prozessdaten mit Lerntagebüchern reichen. Alle vorgestellten Studien wurden in realen Lehr-Lern-Settings durchgeführt und weisen dadurch eine hohe praktische Relevanz auf. Allerdings gehen damit auch methodische Einschränkungen einher: Nicht immer ist es möglich, eine Kontrollgruppe zu rekrutieren, manchmal können wichtige Aspekte doch ,nur“ mit Fragebögen erfasst werden und DropOuts lassen sich bei längsschnittlich angelegten Datenerhebungen nicht vermeiden.

Dennoch heben die Beiträge in der Gesamtschau die Bedeutung von Selbstregulationskompetenzen für unterschiedliche Aufgabentypen sowie Lernsituationen hervor und geben wichtige Hinweise für die Gestaltung von Interventionen zur Förderung des selbstregulierten Lernens. Sie zeigen, dass (1) sich verschiedene Selbstregulationskompetenzen fördern lassen, wobei (2) die individuellen Eingangsvoraussetzungen der Lernenden im selbstregulierten Lernen sowie (3) die internalen Faktoren, die diese beeinflussen, beachtet werden sollten. Zusätzlich machen die Beiträge deutlich, dass (4) mit gezielten Interventionen die Transferleistungen unterstützt werden können. Schließlich weisen die Beiträge darauf hin, dass (5) die Kompetenzen im selbstregulierten Lernen in unterschiedlichen Phasen des Bildungsverlaufs eine wichtige Rolle im Hinblick auf den Lernerfolg und den Verbleib in der Bildungsinstitution einnehmen. Damit verdeutlichen sie, dass dem Erwerb und der Förderung des selbstregulierten Lernens von der Schule bis ins Studium Beach- 
tung zu schenken ist. Gleichzeitig weisen sie auf den Bedarf an weiterer Forschung hin, die sich auch mit den Fragen danach befassen sollte, wie es gelingen kann, eine Förderung des selbstregulierten Lernens in Lehr-Lernkontexten an Schule und Hochschule fest zu verankern und über welche Kompetenzen Lehrende in verschiedenen Bildungsinstitutionen für spezifische Lernaufgaben und -situationen hierfür verfügen müssten.

\section{Literatur}

Bidjerano, T., \& Dai, D. Y. (2007). The relationship between the big-five model of personality and selfregulated learning strategies. Learning and Individual Differences, 17(1), 69-81. https://doi.org/10. 1016/j.lindif.2007.02.001.

Dent, A.L., \& Koenka, A.C. (2016). The relation between self-regulated learning and academic achievement across childhood and adolescence: a meta-snalysis. Educational Psychology Review, 28(3), 425-474. https://doi.org/10.1007/s10648-015-9320-8.

Dignath, C., \& Büttner, G. (2008). Components of fostering self-regulated learning among students. A meta-analysis on intervention studies at primary and secondary school level. Metacognition and Learning, 3(3), 231-264. https://doi.org/10.1007/s11409-008-9029-X.

Donker, A.S., de Boer, H., Kostons, D., Dignath van Ewijk, C.C., \& van der Werf, M.P.C. (2014). Effectiveness of learning strategy instruction on academic performance: a meta-analysis. Educational Research Review, 11, 1-26. https://doi.org/10.1016/j.edurev.2013.11.002.

Engelschalk, T., Steuer, G., \& Dresel, M. (2016). Effectiveness of motivational regulation: Dependence on specific motivational problems. Learning and Individual Differences, 52, 72-78. https://doi.org/10. 1016/j.lindif.2016.10.011.

Hattie, J. A., Biggs, J. A.C., \& Purdie, N. (1996). Effects of learning skills internventions on student learning: a meta-analysis. Review of Educational Research, 66(2), 99-136.

Malmberg, J., Järvenoja, H., \& Järvelä, S. (2013). Patterns in elementary school students' strategic actions in varying learning situations. Instructional Science, 41(5), 933-954. https://doi.org/10.1007/s11251012-9262-1.

McCabe, J. (2011). Metacognitive awareness of learning strategies in undergraduates. Memory and Cognition, 39(3), 462-476. https://doi.org/10.3758/s13421-010-0035-2.

Moos, D. C., \& Ringdal, A. (2012). Self-Regulated Learning in the Classroom: A Literature Review on the Teacher's Role. Education Research International, 2012, 1-15. https://doi.org/10.1155/2012/423284.

Paris, S. G., \& Paris, A. H. (2001). Classroom Applications of Research on Self-Regulated Learning. Educational Psychologist, 36(2), 89-101. https://doi.org/10.1207/s15326985ep3602_4.

Perry, N.E., \& VandeKamp, K.J.O. (2000). Creating classroom contexts that support young children's development of self-regulated learning. International Journal of Educational Research, 33(7-8), $821-843$.

Peverly, S. T., Brobst, K. E., Graham, M., \& Shaw, R. (2003). College adults are not good at self-regulation: a study on the relationship of self-regulation, note taking, and test taking. Journal of Educational Psychology, 95(2), 335-346. https://doi.org/10.1037/0022-0663.95.2.335.

Pressley, M., Borkowski, J.G., \& Schneider, W. (1989). Good information processing: what it is and how education can promote it. International Journal of Educational Research, 13(8), 857-867.

Richardson, M., Abraham, C., \& Bond, R. (2012). Psychological correlates of university students' academic performance: a systematic review and meta-analysis. Psychological Bulletin, 138(2), 353-387. https://doi.org/10.1037/a0026838.

Schiefele, U., \& Pekrun, R. (1996). Psychologische Modelle des fremdgesteuerten und selbstgesteuerten Lernens. In F.E. Weinert (Hrsg.), Psychologie des Lernens und der Instruktion. Enzyklopädie der Psychologie (Bd. 2, S. 249-278). Göttingen: Hogrefe.

Sontag, C., \& Stoeger, H. (2015). Can highly intelligent and high-achieving students benefit from training in self-regulated learning in a regular classroom context. Learning and Individual Differences, 41, 43-53. https://doi.org/10.1016/j.lindif.2015.07.008.

Stebner, F., Schuster, C., Dicke, T., Karlen, Y., Wirth, J., \& Leutner, D. (im Druck). The effects of selfregulation training on self-regulated learning competencies and cognitive load: Does the socioeconomic status matter? In J. Sweller, S. Tindall-Ford, \& S. Agostinho (Hrsg.), Advances in Cognitive Load Theory: Rethinking Teaching. Abingdon: Routledge. 
Wolters, C. A., \& Hussain, M. (2015). Investigating grit and its relations with college students' self-regulated learning and academic achievement. Metacognition and Learning, 10(3), 293-311. https://doi. org/10.1007/s11409-014-9128-9.

Zimmerman, B. J. (2000). Attaining Self-Regulation. A social cognitive perspective. In M. Boekaerts, P. R. Pintrich, \& M. Zeidner (Hrsg.), Handbook of self-regulation (S. 13-39). San Diego, CA: Academic Press.

Zimmerman, B.J., \& Schunk, D.H. (Hrsg.) (2011). Handbook of self-regulation of learning and performance. New York, NY: Taylor \& Francis. 